\title{
APUESTAS DE ALTO RIESGO: \\ SU IMPORTANCIA PARA LA REGULACION FINANCIERA
}

\author{
Mauricio Naranjo González ${ }^{1}$ \\ JuLıO, 1996 \\ DOCUMENTO de InVESTIGACIÓN No. 9603 \\ DiRECCIÓN GeNeRAL de InVESTiGACIÓN ECONÓMICA
}

BANCO de MÉXICO

\footnotetext{
${ }^{1}$ Investigador económico, Banco de México. Agradezco los comentarios de George Akerlof, Agustín Carstens, Matthew Rabin, Paul Romer y James Wilcox, así como la valiosa ayuda de Alexis Milo Caraza. Las opiniones contenidas en este documento representan los puntos de vista del autor y no necesariamente aquéllos del Banco de México.
} 


\title{
1 INTRODUCCIÓN
}

2 LOS JUEGOS DE TODO O NADA

\section{EL MODELO}

3.1 EL JUEGO AGENTE-PRINCIPAL

3.2 LA DECISIÓN DEL GANADOR

4. DESPERTANDO DE LA PESADILLA

5. DISCUSIÓN

6. CONCLUSIONES

ANEXO 1

BIBLIOGRAFÍA

\author{
Lista de Gráficas
}

Esquema 1: La apuesta del banquero I

Esquema 2: La apuesta del banquero II

Esquema 3: La apuesta del banquero III

Esquema IV: La apuesta del banquero IV

Esquema V: La apuesta del banquero V

\section{Lista de Cuadros}

Tabla 1: Negocios y Entretenimiento

Tabla 2: Deportistas mejor pagados 


\title{
APUESTAS DE ALTO RIESGO: SU IMPORTANCIA PARA LA REGULACION FINANCIERA
}

\author{
Mauricio Naranjo González \\ JULIO, 1996
}

\section{RESUMEN}

Las apuestas de alto riesgo, o de todo o nada, aparecen como una conducta recurrente en los mercados en que el ganador se lleva todo. Esta actitud de gran propensión al riesgo puede ser explicada por la búsqueda de status y una percepción incorrecta de la probabilidad de fracaso. Su análisis es relevante para la regulación financiera, ya que las apuestas de alto riesgo son una causa potencial de riesgo sistémico. Este documento diseña la regulación de apuestas de alto riesgo en el sistema financiero a través de un mecanismo de "destrucción de recursos".

\section{InTRODUCCIÓN}

Imaginese que acaba de comprar un banco. Mejor aún, dos bancos y enfrenta la siguiente decisión: consolidar su conglomerado financiero o - aprovechando su racha de buena suerte - apostar toda su riqueza a la posibilidad de convertirse en una de las cinco personas más ricas del país. La mayoría optaría por la primera alternativa. La lógica de esta decisión se basa en suponer que la situación económica personal ya no es problema, al ser dueño de dos bancos, por lo que es mejor consolidar dicha posición que entrar en una apuesta de alto riesgo, la cual en términos de consumo, no mejoraría la situación, o lo haría sólo marginalmente. No obstante lo anterior, muchos agentes económicos, cuando 
enfrentan esta disyuntiva, prefieren apostar su riqueza, fenómeno que por extraño que parezca, se repite continuamente en muchos países con graves consecuencias en los mercados financieros. ${ }^{2}$

¿Por qué alguien con una vida confortable, sólidamente establecido como un rico y exitoso hombre de negocios, arriesga todo por la oportunidad de convertise en el hombre más acaudalado del país? Parece un caso de ambición patológica; ¿cuál es la utilidad de tener una casa de vacaciones más si ya se poseen cinco?; ¿jugar una apuesta de todo o nada es una decisión correcta para el típico agente económico adverso al riesgo?

Este documento contesta estas preguntas e intenta explicar porqué se toman apuestas de alto riesgo que a primera vista parecen irracionales. El análisis muestra la relevancia que la propensión al riesgo guarda en el diseño de políticas y particularmente en la regulación financiera.

La siguiente sección abunda sobre los conceptos de apuestas de todo o nada y de propensión al riesgo. La tercera sección desarrolla un modelo en el cual un individuo opta por una apuesta de alto riesgo, en este caso, una estrategia óptima en un mercado donde el ganador se lleva todo. La cuarta parte del documento analiza la regulación de los juegos de todo o nada y explica el porqué la conducta de un agente amante del riesgo es un problema potencial para el regulador. Al final se presentan las conclusiones, junto a una propuesta para la regulación del mercado financiero.

\section{Los Juegos de TODO o NADA}

El comportamiento de un agente económico amante del riesgo, por ejemplo un banquero que participa en una apuesta de alto riesgo en la que podría perder todo o bien recibir una gran ganancia, no tiene mucho sentido desde la perspectiva económica del consumo. Una apuesta de alto riesgo es una apuesta asumida por un agente económico que tiene dos posibles resultados: un gran pago o una enorme pérdida, en la cual el agente pierde inclusive sus recursos iniciales. Incluso, el juego puede tener un rendimiento esperado negativo en términos de consumo, a pesar de lo cual el agente hace la apuesta.

Tomar esta apuesta es irracional a menos de que el rendimiento esperado sea positivo, para lo cual se requiere que el pago sea muy grande, o bien que la probabilidad del resultado malo sea muy pequeña. El argumento principal de esta investigación es que los agentes perciben una probabilidad de fracaso muy pequeña y por el contrario, un gran beneficio de resultar ganadores. Estas percepciones pueden ser falsas. Por tanto, el comportamiento de un jugador ante un riesgo de todo o nada se explica mejor a partir de la propensión al riesgo de los agentes, que en términos de una alta utilidad esperada por consumo, del tipo Von Neumann-Morgenstern.

Los juegos de todo o nada no son excepcionales. En los mercados financieros tenemos muchos ejemplos recientes: Ivan Boesky y Michael Milken de Drexel Burnham Lambert Inc., cuando desarrollaron el mercado americano de bonos chatarra durante los años ochenta; ${ }^{3}$ Mario Conde de Banesto en España en 1993; Clifford Clark y Robert Altman del Bank of Credit \& Commerce International SA. en 1991; los hermanos Cisneros en Venezuela y Carlos Cabal en México, ambos casos en 1994. Todos ellos eran exitosos y ricos financieros, a pesar de lo cual estuvieron inmiscuidos en fraudes, en el intento de lograr que sus corporaciones financieras adquirieran una posición dominante en sus respectivos mercados.

En deportes, actividad donde la posición relativa de una persona es más clara y relevante, también encontramos casos de tomadores de altos riesgos, dispuestos a convertirse en el ganador. El canadiense Ben Johnson usó drogas ilegales al competir en los Juegos Olímpicos de 1988 . Diego Armando Maradona, en algún tiempo el mejor jugador de fútbol soccer en el mundo, usó drogas prohibidas durante la Copa del Mundo 1994. En patinaje artístico sobre hielo, Tonia Harding aparentemente conspiró para lesionar a su competidora más cercana antes de los Juegos Olímpicos de Invierno 1994.

\footnotetext{
${ }^{2}$ Los incentivos perversos como los descritos se pueden generar por la existencia de información asimética y seguro de depósitos, aspectos que han sido ampliamente analizados en la literatura.

${ }^{3}$ Milken y Boesky permanecieron dos años en prisión, pero pudieron conservar los beneficios obtenidos en el mercado de bonos chatarra. Ciertamente perdieron la apuesta pero no obtuvieron un pago nulo o negativo. Sin embargo, si consideramos que dos años en cárcel no están compensados por US\$500 millones, entonces su comportamiento sí fue una apuesta de alto riesgo.
} 
En política, que se podría considerar como el mercado extremo donde el ganador se lleva todo, se asumen altos riesgos para ganar una elección. Un buen ejemplo es el Presidente Richard Nixon, quien durante su campaña de reelección en 1972 mandó realizar riesgosas acciones de espionaje que buscaban otorgarle un margen extra para ganar la elección. La apuesta se convirtió en pérdida cuando el espionaje se hizo público, y Nixon fue forzado a renunciar dos años después.

En sociología criminalística, esta conducta ha sido llamada crimen de "cuello blanco", a partir de la contribución de Sutherland en 1940. Sutherland nunca dio una definición del crimen de "cuello blanco"; lo más cercano fue hablar de "la respetabilidad y el alto rango social" del criminal, "una persona de la alta clase socioeconómica", quien "viola las leyes diseñadas para regular sus actividades de trabajo" (Ver Geis [1992]). Ciertamente, lo que llamamos apuestas de alto riesgo son parte de los crímenes de "cuello blanco" estudiados en sociología (ver Braithwhite [1992] para algunos ejemplos). El análisis en este documento va más allá en varios aspectos que el de Sutherland y otras contribuciones previas en sociología. Primero, las apuestas de todo o nada son caracterizadas como un tipo especial de crímenes de "cuello blanco". Segundo, se identifica la motivación del jugador en términos de status. Y tercero, ofrecemos un mecanismo regulatorio.

En economía sólo se han analizado las apuestas de alto riesgo de una manera superficial. Allais (1953) planteó una famosa paradoja económica donde los agentes tienen que escoger entre dos apuestas, en donde una de ellas tiene la tentación de una gran ganancia. Su elección viola los supuestos de la teoría de utilidad esperada. Más recientemente, Kahneman y Tversky (1979), inspirados por evidencia psicológica, demostraron que las funciones de utilidad no son siempre cóncavas, es decir, que las personas en algunos casos son amantes del riesgo. Las apuestas de alto riesgo en mercados en que el ganador se lleva todo son un caso específico en que la utilidad esperada Von Neumann-Morgenstern no explica el comportamiento de los agentes. El propósito de este trabajo es - a partir de las aportaciones de Allais, Kahneman y Tversky — analizar el comportamiento económico en este caso específico.

La propensión al riesgo aparece como una actitud recurrente, a pesar de que usualmente sólo estamos conscientes de aquellos juegos cuyo resultado es malo. Sin un mal resultado, el banquero amante del riesgo seguiría siendo un respetable banquero y no nos enteraríamos de su comportamiento.

Las apuestas de alto riesgo son relevantes en la regulación financiera, en virtud de que en estas situaciones las preferencias de la sociedad y las del jugador son diferentes. En un mercado del tipo el ganador se lleva todo, una estrategia de alta varianza puede ser óptima para el agente. Por otro lado, el trabajo del regulador en este juego es evitar una apuesta de alto riesgo. Para ilustrar el comportamiento en este tipo de apuestas y subrayar el papel del regulador financiero, construimos el juego a partir de la historia del banquero.

El propósito principal del documento es analizar las apuestas de alto riesgo realizadas por los agentes financieros; sin embargo, es posible aplicar el modelo a otras decisiones de apuesta, en las que el regulador sería sólo otra autoridad. Este trabajo afirma que una importante motivación para asumir el riesgo en los mercados donde el ganador se lleva todo es la probabilidad del jugador de subir a la cima de la escala social. La propensión al riesgo motivada por la ambición de status o posición, unida a la mala apreciación acerca del posible éxito de la apuesta por parte del jugador, también cambian la respuesta óptima del regulador.

\section{EL MOdeLo}

\subsection{El Juego Agente-Principal}

El juego que se plantea es un modelo agente-principal, en el que el regulador (o principal) trata que el agente tome una acción determinada. Los modelos agente-principal se enfocan a resolver el acceso limitado a la información por parte del regulador y pretenden brindar una solución a partir de incentivos. Esta área de la economía que se concentra en el diseño de mecanismos de mercado, surge de Baron y Myerson (1982). Los modelos de agente-principal se concentran en problemas de abuso inmoral (moral hazard) y selección adversa. Por ejemplo, un problema de abuso inmoral dentro de este tipo de modelos es el que se podría dar entre un trabajador (agente) y su jefe (principal). Este último intenta que el 
primero se esfuerce más en su trabajo, pero tiene problemas para supervisarlo. La solución a este tipo de modelos ocurre cuando el trabajador internaliza el resultado de sus acciones ${ }^{4}$ (ver Grossman y Hart [1983]). El problema de selección adversa se presenta cuando el principal no conoce el tipo de agentes que enfrenta, por ejemplo una empresa de seguros que desconoce el riesgo al que están sujetos sus clientes. En consecuencia, aquellos individuos que representan los peores riesgos tienen un mayor incentivo a contratar un seguro. En este caso, la solución se da al establecer incentivos para que los agentes revelen su tipo (ver Rothschild y Stiglitz [1976]). Nuestro modelo ilustra el problema de abuso inmoral entre un regulador financiero y un banquero.

El problema se puede definir por medio de un juego que cuenta con tres etapas en las que de forma sucesiva un banquero y el regulador toman acciones y la naturaleza se manifiesta de cierta manera, lo cual todo en su conjunto determinan un resultado. El modelo empieza con un banquero ( $\mathrm{B}$ ) tomando la decisión entre apostar o no apostar. Si él apuesta, las circunstancias, que llamaremos la naturaleza ( $\mathrm{N}$ ), arrojan un resultado bueno o un resultado malo. Finalmente, la autoridad o regulador ( $R$ ), sin conocer la historia de acciones previas del mismo juego, decide ser estricto o no estricto - donde ser estricto implica un costo (ver esquema 1).

El juego parte del supuesto de que el banquero, por el sólo hecho de poseer el banco, recibe al final un pago de 1. Las magnitudes de los pagos no implican algo sobre la naturaleza del juego, sólo están normalizadas para volver sencillo el ejercicio. El banquero puede asegurar su pago de 1 si no apuesta, o puede jugarse su dotación inicial. Los pagos para el banquero sólo reflejan un resultado básico de finanzas, en el que proyectos con mayor rendimiento esperado tienen también mayor riesgo o varianza.

Por su parte, al regulador le han asignado la tarea de supervisar y monitorear estrictamente al banquero. Se supone que el regulador tiene preferencias que asignan una utilidad negativa al esfuerzo y una positiva al ocio $^{5}$ (Arrow [1963] y Akerlof [1982]). Por tanto, regular al banquero no es fácil, pues el regulador no recibe ninguna utilidad cuando es estricto y desincentiva la mala actitud del banquero. Y por el contrario, recibe una utilidad de 1 cuando no hace su trabajo tan bien como podría. Por último, la acción de la naturaleza captura el azar del juego: el banquero, después de apostar, puede obtener tanto una ganancia como una pérdida, de la misma manera que en un "volado" la naturaleza determina si el resultado es "águila" o "sol".

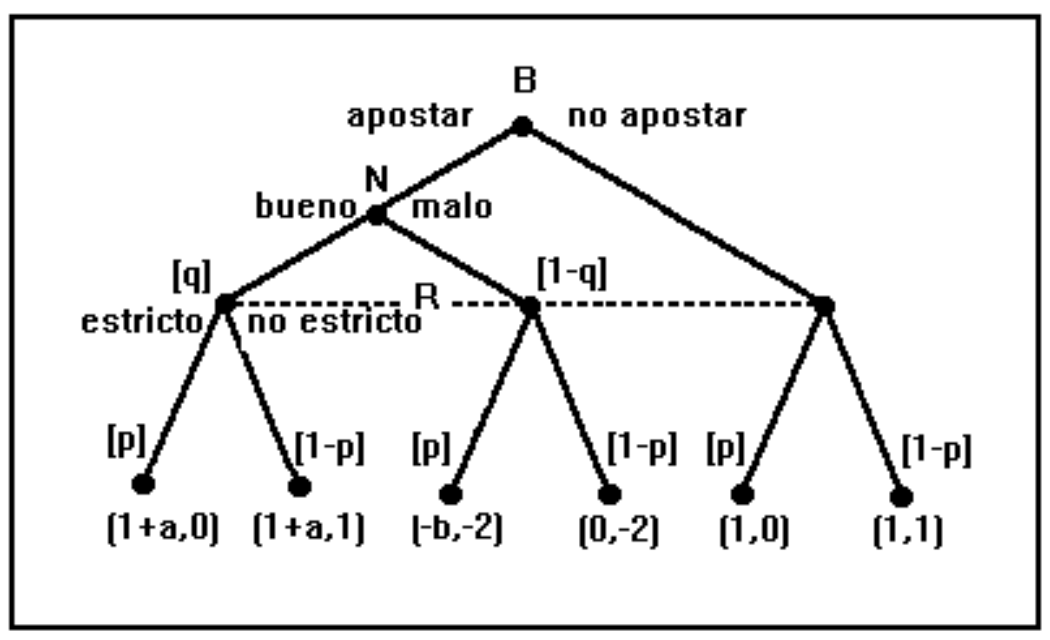

Esquema 1: La apuesta del banquero I

\footnotetext{
${ }^{4}$ Por "internalizar" en este contexto se quiere dar a entender que el trabajador se atiene a las consecuencias de sus acciones.

${ }^{5}$ Ocio aquí se considera como una forma de consumo, de la misma manera que se ha tratado ampliamente en la literatura microeconómica. De ninguna manera el papel que juega el ocio en este modelo es una referencia a características personales del regulador, es sólo un supuesto que permite analizar los incentivos a los que debe estar sujeta la autoridad para garantizar su buen desempeño
} 
Como ya mencionamos, si el banquero no apuesta obtiene un pago de 1, por el sólo hecho de poseer el banco, mientras que el regulador obtiene 1 si no es estricto y 0 si lo es. Si el banquero apuesta y el resultado es bueno, obtiene un pago de $1+a, a>0$, independientemente de la acción que lleve a cabo el regulador, ya que no es sorprendido. Sin embargo, el regulador obtiene 1 si no es estricto y 0 si lo es, ya que su trabajo requiere esfuerzo. Ahora, si el banquero apuesta y el resultado es malo, recibe un pago de 0 si el regulador no es estricto, y de $-b, b>0$ si lo es. Cuando el regulador es estricto, el castigo que le impone al banquero por apostar es $b$. Pero un juego con un resultado malo ocasiona una crisis en el sistema financiero y el regulador obtiene un pago de -2, sea estricto o no. En este escenario, es irrelevante su esfuerzo de ser estricto.

Supongamos que de acuerdo a las expectativas del banquero, la naturaleza estará en un buen estado con una probabilidad $q$, y con una probabilidad $p$ el regulador será estricto. El regulador tradicional tiene dos herramientas de política: $p$ y $b$, la probabilidad de ser estricto y el castigo que impone al banquero cuando éste apuesta y es atrapado. Para que el juego sea interesante, suponemos que $q(1+a)-(1-q) b<1$. Es decir, cuando el regulador es estricto, la mejor respuesta para el banquero es no apostar. Ya que el banquero no sabe si la naturaleza arrojará un resultado bueno o malo, su pago esperado - cuando el regulador es estricto - es $q(1+a)-(1-q) b$ si apuesta y 1 si no apuesta. También suponemos que $q(1+a)>1$, lo que indica que cuando el regulador no es estricto, la mejor respuesta del banquero es apostar. De la misma forma, dado que el banquero no sabe la acción que tomará la naturaleza, su pago esperado - cuando el regulador es no estricto - es $q(1+a)$ si apuesta y 1 si no apuesta. Finalmente, asumimos que $q<\frac{2}{3}$ por sencillez, ya que queremos descartar el caso en que el regulador favorece las apuestas de alto riesgo en virtud de que la probabilidad de un resultado malo es muy pequeña, por lo que la posibilidad de generar una crisis en el sistema financiero es muy baja.

Ser estricto es una estrategia dominada ${ }^{6}$ para el regulador, de modo que el único equilibrio puro de Nash ${ }^{7}$ es (no estricto, apostar). ${ }^{8}$ En la solución de Nash, el regulador obtiene un pago negativo de $q-2(1-q)$, lo cual ex-post es un resultado malo, ya que para él sería mejor comprometerse a ser estricto, $p=1$, y recibir un pago de cero. El análisis del juego se concentra en este compromiso, es decir, en la credibilidad y consistencia intertemporal del mecanismo regulatorio.

Consideremos un ejemplo con $q=\frac{1}{4}, a=4$, y $b=\frac{1}{2}$. Imaginemos que el regulador percibe que el equilibrio de Nash no es una buena solución para él, y que el banquero lo sabe. Ahora el banquero tiene razón en pensar que $p \neq 0$, y su mejor estrategia bayesiana es apostar si $p \leq \frac{2}{3}$ y no apostar en cualquier otro caso. Así, si el regulador es estricto con una probabilidad mayor a $\frac{2}{3}$, el banquero nunca elegirá apostar. Es importante mencionar una interpretación alternativa del juego, que no afecta de forma cualitativa la naturaleza del juego, pero que sí cambia este resultado cuantitativo. Esta interpretación alternativa del juego nos revela la sensibilidad del modelo a los supuestos que estamos considerando.

La intuición de esta nueva interpretación del juego es que el banquero puede ser castigado después de apostar no sólo cuando la naturaleza elige un resultado malo, sino también cuando la naturaleza elige un resultado bueno pero el regulador se percata de que se realizó la apuesta. La caracterización del juego considera que la elección de la naturaleza entre un resultado bueno o malo incluye no sólo que la apuesta obtenga una ganancia o una pérdida, sino también el hecho de que el regulador se percate o no se percate de que se realizó la apuesta. Es decir, en nuestro caso general cuando la naturaleza elige un resultado malo, esto implica que la apuesta resultó en una pérdida, o que la apuesta resultó en una ganancia pero que el regulador detectó la apuesta.

\footnotetext{
${ }^{6}$ Una estrategia dominada es aquella que nunca será elegida de forma racional ya que siempre ofrece un pago menor que la estrategia alternativa.

${ }^{7}$ En teoría de juegos un equilibrio puro de Nash es aquel en el que la estrategia elegida por ambos agentes es óptima dada la estrategia óptima del otro agente.

${ }^{8}$ Nuestro análisis no considera estrategias mixtas, ya que no aportan mayor comprensión a esta discusión. Es decir, los agentes tienen que decidirse por un curso de acción y no pueden tomar acciones de forma aleatoria de acuerdo a cierta probabilidad.
} 
Esta interpretación del juego es la más sencilla y general. No obstante, podríamos separar el hecho de que la naturaleza obtenga un resultado malo o bueno, del hecho de que el regulador detecte o no la apuesta. Esta interpretación alternativa del juego da como solución que el banquero no apostará cuando la probabilidad de que el regulador tome una actitud estricta sea mucho menor que en el caso general para el ejemplo que aquí se considera - obtenemos que $p \leq \frac{1}{7}$, que sustituye el valor anterior de $\frac{2}{3}$.

Aumentar la probabilidad de castigo para el banquero es equivalente a aumentar el castigo (b) lo que resulta en un menor incentivo a apostar. El desarrollo de esta interpretación se presenta en el anexo 1.

El esquema del juego ilustra la naturaleza del problema pero no explica cuáles son los incentivos de las apuestas de alto riesgo. Para ello, necesitamos primero extender el análisis en dos direcciones. Primero, la propensión al riesgo del jugador, el cual puede cambiar el valor de a y/o la percepción de $q$. Segundo, la solución al juego si se juega de forma repetitiva un número infinito de veces. Discutiremos primero la solución al juego repetido y después la propensión al riesgo del jugador.

El problema que el regulador enfrenta no es un juego de una sola vez, especialmente para un regulador financiero. El incentivo de apostar para el banquero en un juego repetido de forma infinita es aún mayor, ya que un número infinito de ganancias repetidas está de por medio. Por esta razón, el regulador tiene que ser aún más estricto si quiere eliminar las apuestas de alto riesgo. ${ }^{9}$ Imaginemos que el regulador puede ser de dos tipos: "racional" en cuyo caso siempre juega estricto, o "irracional" (un regulador flojo) en cuyo caso siempre juega no estricto. Supongamos que el banquero no conoce a qué tipo de regulador se enfrenta, pero intuye que es "racional" con una probabilidad $\varepsilon$. Con $\delta$ como factor de descuento, la mejor decisión para el banquero es jugar si

$$
\varepsilon\left\{\frac{q(1+a)-(1-q) b}{1 / \delta}+\frac{\delta}{1-\delta}\right\}+(1-\varepsilon)\left\{\frac{q(1+a)}{1-\delta}\right\}>\frac{1}{1-\delta}
$$

El lado derecho de esta desigualdad es el pago recibido por escoger siempre no apostar, ya que en este caso es el pago para todos los periodos es de 1. Esta perpetuidad, considerando el factor de descuento $\delta$, es igual a $\frac{1}{1-\delta}$. El lado izquierdo es el pago obtenido por apostar, sólo una vez si el regulador es “ racional", y siempre si el regulador es del tipo "irracional". Si el regulador es "irracional" el banquero siempre apostará y recibirá un pago esperado de $q(1+a)$ en todos los períodos. Mientras que si el regulador es "racional" el banquero apostará sólo el primer período, obtendrá un pago esperado de $q(1+a)-(1-q) b$ y al mismo tiempo se dará cuenta de su tipo. Por tanto, en el resto de los períodos no apostará y recibirá un pago — del período uno en adelante — de 1, lo que descontado es igual a $\frac{\delta}{1-\delta}$.

Para los valores usados en nuestro ejemplo y para un factor de descuento de 0.95 , la mejor respuesta del banquero es apostar si $\varepsilon<0.9767$. Es decir, en realidad la única manera de evitar las apuestas de alto riesgo, bajo un juego repetido, es tener prácticamente con certeza un regulador "racional".

\subsection{La Decisión del Ganador}

En El Jugador de Dostoyevsky el espíritu de juego es claramente expresado por Alexey Ivanovich al entrar al casino:

"Mi certidumbre de ganar puede parecer ridícula; sin embargo, atacar me deja en paz ... Tengo una idea curiosa, sin sentido, de que estoy destinado a ganar en las mesas de juego. No puedo decir por que pienso así, pero así pienso, y con cierta seguridad."

\footnotetext{
${ }^{9}$ La solución al juego repetido un número finito de veces es el mismo que el juego de un sólo período, dado que el equilibrio de Nash siempre es óptimo en el último período. Por tanto, el equilibrio de Nash también es óptimo en el penúltimo período, y así sucesivamente hasta el primer período.
} 
Aun después de perder, Alexey reflexiona:

"Siento un tipo de placer temeroso - el placer del éxito, de conquista, de poder (¿Cómo puedo expresarlo mejor?) ... iAh! ¡En esos momentos uno se olvida de uno mismo y de sus fracasos anteriores! Esto lo he ganado arriesgando mi propia vida. He desafiado el riesgo y observado nuevamente que soy un miembro de la humanidad!"

Esta cita literaria captura dos puntos importantes que constituyen nuestra hipótesis acerca del comportamiento del jugador: 1) a pesar de la probabilidad positiva de un resultado adverso, el jugador percibe que ganará, y 2 ) su motivación para correr el riesgo no es el dinero sino algo más, que llamaremos "status".

El primer punto es que el jugador es excesivamente optimista con respecto a su apuesta. Los límites de procesar información y estimar probabilidades de los agentes económicos han sido discutidos en economía durante largo tiempo, (v.g., por Herbert Simon). Esta investigación sostiene que las apuestas de alto riesgo se realizan, al menos en parte, en virtud de que la probabilidad de perder es percibida erróneamente por el jugador. En términos del juego, podemos decir que el banquero estima equivocadamente $q$.

El error de estimación puede tener al menos tres causas. La primera es una actualización bayesiana; el banquero estimó erróneamente $q$ debido a que históricamente había tenido suerte al asumir riesgos, es decir, sólo había obtenido buenos resultados en las apuestas previas. Los psicólogos han descubierto que el procesamiento humano de información está sujeto a considerables fluctuaciones al evaluar probabilidades, inclusive en decisiones cotidianas de carácter repetitivo (ver Kahneman, Slovic y Tversky [1982 ] y Nisbet y Ross [1980 ]). Es más, lo que esta literatura sugiere - en términos de nuestro modelo - es que la estimación de probabilidades fluctúa en favor de la apuesta del jugador (ver Ainslie [1992 ] y Thaler y Ziemba [1988 ]).

La psicología que se basa en la evidencia que arroja la conducta humana expone una segunda fuente de sesgo en las probabilidades que las personas estiman. Esta explicación parte del hecho de que la gente tiene altas expectativas no realistas de ellos mismos. Ya que la gente promedio tiende a pensar que vivirán más que el promedio, que tendrán menos enfermedades que el promedio de las personas, que correrán con mejor suerte que el promedio, ellos están subestimando sus riesgos. "Existe una propensión ubicua, basada en características biológicas humanas, al optimismo infundado" (Perloff y Fetzer [1986]).

La tercera causa de error en la estimación es la disonancia cognoscitiva, es decir, la tendencia a formar las creencias en función de nuestros deseos o expectativas, a pesar de la evidencia. Los psicólogos han estudiado la disonancia cognoscitiva por largo tiempo y han determinado que es un mecanismo contra la vacilación, una aprehensión realista del costo fundamental de la ambivalencia o la continua reevaluación (ver Ainslie [1992]). Akerlof y Dickens (1982) han aplicado la disonancia cognoscitiva a la economía, afirmando que las personas poseen preferencias sobre sus creencias acerca del estado que guarda el mundo, tienen cierto poder para cambiar las creencias que adoptan y estas creencias persisten una vez elegidas. En nuestro contexto, la disonancia cognoscitiva implica que si el banquero ya realizaba negocios financieros asumiendo riesgos, tendería a subestimar el riesgo de apostar, i.e. a sobrestimar $q$. Una vez que tomó el riesgo inicial, pudo haber creído que las apuestas en el mercado financiero no eran realmente tan riesgosas, lo que podría haberlo ayudado a mantener la calma, pero al mismo tiempo tal vez lo hizo aumentar su apuesta.

La disonancia cognoscitiva también actúa en la probabilidad esperada de castigo. Quizá el banquero pensó que, de ser sorprendido, no sería sancionado; después de todo, él era un influyente hombre de negocios, respetado inclusive por funcionarios del gobierno. De acuerdo con Sutherland (1940), las motivaciones individuales del criminal de "cuello blanco" son la respetabilidad, la posición laboral, el acceso a la riqueza y al poder, mismas que crean estructuras de oportunidad únicas para el crimen. La creación de estructuras de oportunidad para el crimen - la posición social y los contactos con las autoridades legales - son relevantes para entender las diferencias en la aplicación de las leyes. El banquero pudo haber tenido buenas razones para pensar que las posibilidades de ser perseguido eran 
tenues. Shapiro (1984) aporta evidencia de que la Securities and Exchange Commision sólo canaliza el diez por ciento de los fraudes a través de sanciones criminales, mientras que los restantes son manejados a través de soluciones civiles o administrativas. Coleman (1989) ofrece amplia evidencia con respecto al diferente trato que la élite criminal recibe en el sistema legal.

Como se mencionó, la segunda hipótesis de nuestro análisis de apuestas de alto riesgo es que la motivación para tomar un riesgo no es el dinero sino el status. Tradicionalmente, el agente económico es representado como adverso al riesgo. Sin embargo, hay casos en que muchos agentes económicos asumen grandes riesgos, y no siempre con la expectativa de una gran recompensa. En el resto del capítulo, presentamos una explicación de la propensión al riesgo en términos de un comportamiento económico.

La propensión al riesgo fue formalmente analizada en economía por Friedman y Savage (1948), aunque ya había sido estudiada antes por John Stuart Mill, entre otros. Friedman y Savage sostenían que la función de utilidad de un agente económico era cóncava por abajo de su nivel de ingreso y convexa por encima de éste. Bajo este esquema, la aversión al riesgo es solamente una condición local de la función de utilidad, mientras que la propensión al riesgo es una conducta consistente para un agente económico. El análisis de Friedman y Savage es muy importante porque reconoce la búsqueda de riesgo como un hecho económico. Sin embargo, se ha demostrado que su hipótesis acerca de la función de utilidad es errónea. Kahneman y Tversky (1979), con mucha mas evidencia, encontraron que la función de utilidad depende de cambios relativos en el ingreso y que es cóncava por encima del nivel de ingreso del agente - el punto de referencia - y convexa por abajo de él.

La propensión al riesgo y su motivación, en presencia de un pago esperado negativo, es una pregunta que la teoría económica no ha podido resolver. Algunas disciplinas distintas de la economía ofrecen algunas respuestas. La biología sostiene que en términos generales los machos se caracterizan por su propensión al riesgo, ya que en su competencia reproductiva, las posibilidades de obtener una pareja hembra y de ese modo perpetuarse, son mayores para los ganadores o los miembros más sobresalientes del grupo. En virtud de que la recompensa por ser un ganador es grande, existe un gran incentivo por tratar de serlo, lo que vuelve óptima una estrategia de riesgo (ver Daly y Wilson [1983]).

En los humanos, la propensión al riesgo es vista como una conducta evolutiva, un mecanismo de respuesta al medio que enfrentaba un ámbito natural lleno de peligros. "Ningún riesgo, ninguna ganancia debió haber sido una regla primordial; $y$ en donde arriesgarse significaba exponerse a grandes riesgos: fuego, alturas, frío, hambre, depredadores, enemigos humanos" (Konner [1990]). Racionalidad limitada y propensión al riesgo fueron seleccionadas como la conducta humana óptima en una época donde la esperanza de vida era de treinta años, por lo que resulta que actualmente estamos equipados con un sistema para evaluar riesgos que quizá no es el adecuado para responder con eficiencia al medio ambiente moderno.

Nuestro análisis nos lleva a considerar una función de utilidad no convencional que dependa no sólo de remuneraciones tangibles y a argumentar en contra de la teoría de la utilidad esperada. Este enfoque no es nuevo en economía, algunos ejemplos incluyen: Kahneman y Tversky (1979) que considera los cambios relativos en ingreso y consumo como una manera de evaluar el riesgo; Akerlof (1982) y Rabin (1993) que toman en cuenta los elementos de justicia; y Romer (1994) que considera el efecto de las promesas y las venganzas. Como Ainslie (1992) apunta, tiene sentido considerar una función de utilidad más amplia, ya que sólo una pequeña parte de las decisiones humanas se toman sobre bienes o asuntos de carácter monetario.

La función de utilidad de nuestro análisis considera el status, a partir del hecho de que las personas no se preocupan sólo de sus pagos monetarios sino también de su posición social. El mismo rendimiento monetario puede ser más o menos atractivo, dependiendo de la posición económica que éste incluya. Frank (1985), usando una gran cantidad de evidencia psicológica, introdujo formalmente la idea de la posición relativa en economía, sosteniendo que estamos inmersos en una competencia posicional y que nuestro lugar en la pirámide económica es para todos muy significativo y de alta relevancia.

La temeridad tiene una explicación adicional si consideramos el status que una acción riesgosa puede ofrecer. Por esta razón esta investigación sostiene que una motivación importante para realizar una 
apuesta de alto riesgo es precisamente la oportunidad de alcanzar un mayor status. Bajo este enfoque, el modelo tiene que ser replanteado: la motivación del banquero para apostar es ahora no sólo la recompensa monetaria sino la posición social que podría alcanzar con la apuesta. Lo que denotamos como a en el modelo es aún más grande si incluimos el ascenso en la escala social. De este modo, las apuestas no son sólo una actividad económica, sino que sirven como un elevador en la estructura de posiciones.

Observando nuestro juego de apuestas, debemos notar que el resultado es muy sensible a la magnitud de a; conforme a se incrementa, cuando el banquero apuesta y el regulador es estricto, el castigo se vuelve inefectivo para disuadir al apostador. A medida que a aumenta, el equilibrio de Nash (no estricto, apuesta) se convierte en un equilibrio bayesiano perfecto. ${ }^{10}$ El mecanismo regulatorio será efectivo en disuadir las apuestas sólo en función del status potencial obtenido con éstas, y de la importancia que el status tenga para el jugador.

La importancia que la gente da al status puede funcionar como un mecanismo para ajustarse a un pago esperado de largo plazo. En los mercados donde el ganador se lleva todo, el gran premio de estar en la cúspide, se convierte en una preocupación legítima. Rosen (1981) llamó a esto el "fenómeno de las superestrellas", donde en ciertos tipos de actividad económica hay una gran concentración de recursos entre unos cuantos individuos, marcada tendencia a que la distribución del ingreso no sea uniforme y que existan grandes recompensas en la cima. Los mercados del tipo el ganador se lleva todo parecen tener una importancia creciente en el mundo moderno, y se han propagado a medida que las tecnologías de rendimientos crecientes a escala - comunicaciones, información y sistemas electrónicos - se han extendido. Hay ejemplos notables en el deporte, entretenimiento, publicaciones, administración empresarial, inversión financiera, servicios legales y en la industria de computación tanto en hardware como en software, (ver Frank y Cook [1992]). En todos estos mercados, los principales participantes reciben una gran ganancia por el pequeño desempeño extra que les permite vencer a sus competidores. De forma que el incentivo a ser el ganador afecta el comportamiento de los agentes económicos. Las tablas 1 y 2 muestran las recompensas para los diez mayores jugadores en cuatro mercados del tipo el ganador se lleva todo: ejecutivos y negocios en Estados Unidos, así como entretenimiento y deportes en América del Norte.

\section{Ejecutivos \\ Salario Total 90-94 (Millones)

EUA
$\begin{gathered}\text { Riqueza Neta en } 1995 \\ \text { (Miles de Millones) }\end{gathered}$ \\ Entretenimiento \\ Ganancias 1993-94 (Millones) (Miles de Millones)}

1. Michael Eisner (Disney) 235

2. Sanford Weill (Travelers) 152

3. J.F. O'Reilly (HJ Heinz) 119

4. S. Hilbert (Conseco) 89

5. Bernard Schwartz (Loral) 65

6. H. Solomon (F. Labs) 62

7. L. Coss (G.T. Financial) 55

8. R. Goizueta (Coca Cola) 52

9. W. Sanders III (A. Micro) 50

10. Stephen Wynn (Mirage) 45
1. William Gates III

2. Warren Edward Buffet

3. John Werner Kluge

5. Richard Marvin Devos

6. Jay Van Andel

7. Samuel Newhouse Jr.

8. Donald Newhouse

9. Helen Walton

10. Jim C. Walton
4. Paul G. Allen
15.0

12.0

6.7

6.1

4.3

4.3

4.3

4.3

4.3

4.3
1. Steven Spielberg

2. Oprah Winfrey

3. Barney

4. Pink Floyd

5. Bill Cosby

6. Barbra Streisand

7. Eagles

8. David Copperfield

9. Rolling Stones

10. Harrison Ford
335

105

84

82

60

57

56

55

53

44

Cifras en Dólares

Fuente: Forbes, 1995 varias ediciones.

Tabla 1: Negocios y Entretenimiento

La biología explica la propensión al riesgo de los machos por la distribución de las hembras hacia los miembros de más alto rango en el interior del grupo. Las oportunidades reproductivas son mercados

${ }^{10}$ Es decir, que nueva información no cambia la estrategia óptima de los jugadores. 
donde el ganador se lleva todo, de modo que el riesgo que asumen los machos es una respuesta óptima al tipo de competencia. En la misma forma, el mercado de negocios parece ser un mercado en donde el ganador se lleva todo, por lo que las apuestas de alto riesgo pueden justificarse en el largo plazo. Después de todo, ser el hombre de negocios más rico de EUA es casi tres veces más rentable que ser el décimo y los beneficios de reconocimiento social en términos de status pueden ser aún mayores. Este análisis ofrece una nueva interpretación de la máxima de Gordon Gekko: "La voracidad es buena" ("Greed is good", de la película Wall Street, Oliver Stone [1988]).

El efecto de un mercado del tipo el ganador se lleva todo en la toma de riesgos y la consideración del status puede también explicar por qué la gente juega a la lotería y sobrestima apuestas que no son favoritas (ver Asch y Quandt [1987] para apuestas competitivas y a Thaler y Siemba [1988] para un estudio del comportamiento del apostador). Un billete de lotería y una apuesta no favorita son ambas sobrestimadas - bajo una análisis de retorno esperado - porque ganar el premio mayor implica un cambio en la posición social. Este hecho no se presenta en las apuestas favoritas, las cuales ofrecen, en el mejor de los casos, sólo dos veces su inversión. La teoría económica aun no ha podido explicar por qué la gente hace apuestas que tienen un retorno esperado negativo; sin embargo, la posibilidad de convertirse en el hombre más rico del país, con sus oportunidades implícitas, bien pueden valer el precio.

Lo atractivo de las recompensas en los mercados del tipo el ganador se lleva todo ofrece una explicación de la importancia dada al status y una racionalidad para la conducta de búsqueda de riesgo. Las apuestas de alto riesgo son un comportamiento esperado de los individuos en su intento por llegar a la cumbre. Fuerzas evolutivas hacen que las personas vengan al mundo con un mecanismo que los impulsa a vencer sobre los otros cuando se compite por recursos importantes. La respuesta de los individuos ante el riesgo es un mecanismo muy útil para luchar y sobrevivir en un medio ambiente natural, no obstante en el mundo moderno este comportamiento causa un nivel máximo de desorden. En la siguiente sección se estudian las soluciones regulatorias a los problemas que las apuestas de alto riesgo desatan en el mercado financiero.

\begin{tabular}{|c|c|c|c|c|c|}
\hline Baloncesto & & \multicolumn{2}{|l|}{ Automovilismo } & \multicolumn{2}{|l|}{ Boxeo } \\
\hline \multicolumn{2}{|c|}{ Ganancias en 1994 (millones) } & \multicolumn{2}{|c|}{ Ganancias en 1994 (millones) } & \multicolumn{2}{|c|}{ Ganancias en 1994 (millones) } \\
\hline 1. Michael Jordan & 30.0 & 1. Gerhard Berger & 13.5 & 1. Michael Moorer & 12.1 \\
\hline 2. Shaquille $O^{\prime} \mathrm{Neal}$ & 16.7 & 2. Nigel Mansell & 11.3 & 2. Evander Holyfield & 12.0 \\
\hline 3. Charles Barkley & 9.3 & 3. Jean Alesi & 8.0 & 3. George Foreman & 8.5 \\
\hline 4. David Robinson & 8.4 & 4. Emerson Fittipaldi & 7.5 & 4. Julio C. Chávez & 8.5 \\
\hline 5. Patrick Ewing & 6.0 & 5. Michael Schumacher & 6.0 & 5. Lennox Lewis & 8.3 \\
\hline \multirow{2}{*}{\multicolumn{2}{|c|}{$\begin{array}{l}\text { Tenis } \\
\text { Ganancias en } 1994 \text { (millones) }\end{array}$}} & \multicolumn{2}{|l|}{ Golf } & \multirow{2}{*}{\multicolumn{2}{|c|}{$\begin{array}{c}\text { Futbol Americano } \\
\text { Ganancias en } 1994 \text { (millones) }\end{array}$}} \\
\hline & & Ganancias en 1994 (mi & ones) & & \\
\hline 1. Andre Agassi & 11.4 & 1. Jack Nicklaus & 14.8 & 1. Joe Montana & 10.3 \\
\hline 2. Pete Sampras & 10.6 & 2. Arnold Palmer & 13.6 & 2. Scott Mitchell & 6.4 \\
\hline 3. Steffi Graf & 8.0 & 3. Greg Norman & 8.8 & 3. Heath Shuler & 6.3 \\
\hline 4. Boris Becker & 7.0 & & & 4. Marshall Faulk & 6.2 \\
\hline \multirow[t]{2}{*}{ 5. Stefan Edberg } & 6.5 & & & 5. Dan Wilkinson & 6.1 \\
\hline & & \multicolumn{4}{|c|}{$\begin{array}{l}\text { Cifras en Dólares } \\
\text { Fuente: Forbes, } 1994 \text { varias ediciones }\end{array}$} \\
\hline
\end{tabular}

Tabla 2: Deportistas mejor pagados

\section{Despertando de la Pesadilla}

La pesadilla para el regulador comienza cuando, a pesar de que el apostador enfrenta un pago esperado negativo, el agente sigue adelante y hace una apuesta de todo o nada. Este evento tiene relevancia 
especial en mercados en los que una apuesta de alto riesgo impone costos a los otros agentes, al regulador y al sistema como un todo, por ejemplo en el sistema financiero. Para evitar este fenómeno, se propone un mecanismo para prevenir apuestas de alto riesgo, mediante la destrucción de recursos como una forma de alterar el juego. Este mecanismo también ilustra el carácter general que la regulación de las apuestas de alto riesgo requiere.

La destrucción de recursos, quema de dinero (burning-money), o el uso de la inducción adelantada (forward induction) para afectar la conducta del agente es un concepto de teoría de juegos, presentado por Van Damme (1988). El problema para el regulador proviene de preferir no ser estricto, ya que le disgusta el esfuerzo que implica ser duro. La intención de destruir recursos es volver al regulador indiferente entre ser o no ser estricto. Una vez que el agente está consciente de esta nueva variable, la naturaleza del juego es alterada y, si analizamos hacia adelante el juego, el agente decide no apostar.

Consideremos el juego del esquema 2, una extensión de nuestro juego inicial. El regulador tiene la elección entre mover primero y afirmar que será riguroso, o no hacer ninguna afirmación. Para que la afirmación sea creíble debe implicar un costo; imaginemos que el regulador, al afirmar que será riguroso, destruye el pago que obtendría por no ser estricto, por ejemplo, se compromete a nunca tomar vacaciones. Esta decisión, podría convertirse en una obligación legal para el regulador, por ejemplo tener siempre la misma carga de trabajo o ejecutar un numero fijo de auditorias. ${ }^{11}$

Si el agente afirma su intención de ser riguroso, entonces el subjuego agente-principal puede ser presentado en una forma normal, como en el esquema 3. Este juego tiene dos equilibrios puros de Nash: (no estricto, apuesta) y (estricto, no apuesta). Como en cualquier juego con dos equilibrios de Nash, estamos en presencia de un problema de coordinación. Para el banquero es racional no apostar, porque la afirmación del regulador de ser riguroso actúa como una señal. Si el regulador invierte en hacer una afirmación de ser riguroso, es porque planea mantenerse firme y ser estricto. La solución de Nash seleccionada por el regulador es clara desde el punto de vista de inducción adelantada, ya que al no hacer la afirmación de ser riguroso, el regulador podría asegurar un pago de $q-2(1-q)$. Para el regulador no ser estricto después de destruir sus recursos es una opción irrelevante.

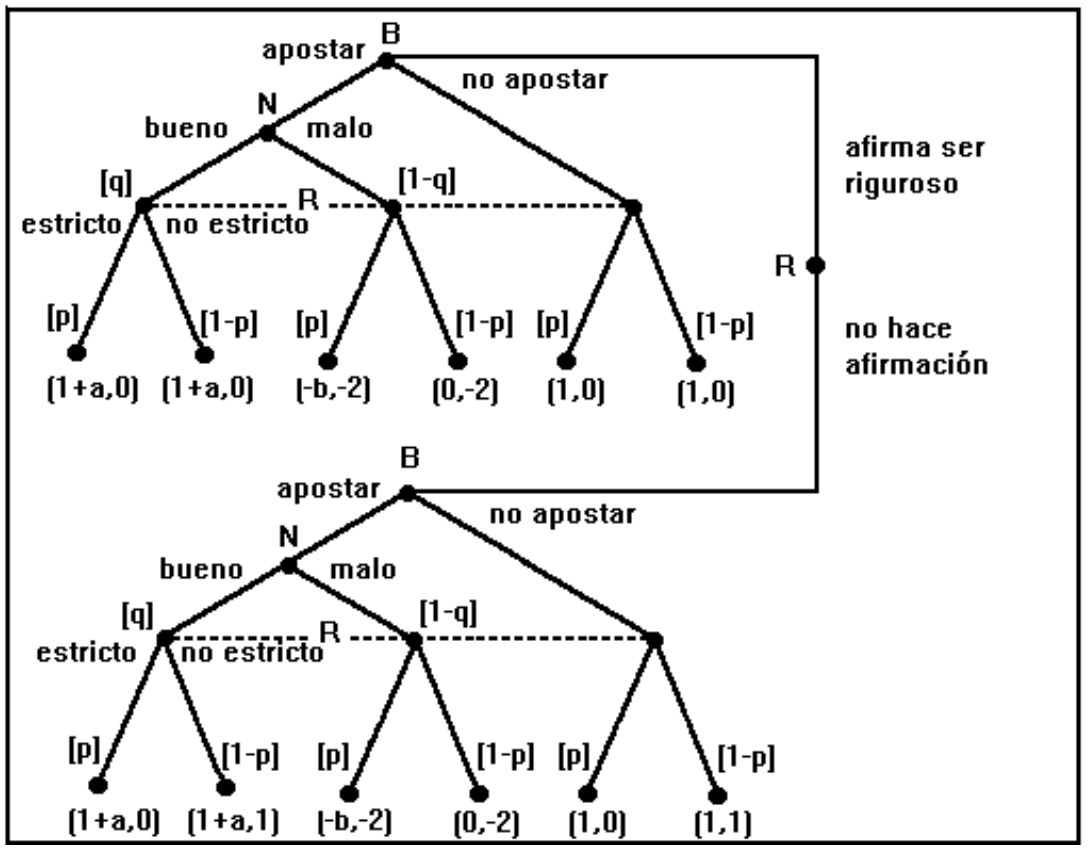

Esquema 2: La apuesta del banquero II

11 Nótese que obtenemos el mismo resultado si asignamos como regulador a una persona adicta al trabajo, o a una persona que disfrute castigando banqueros que apuestan. Un regulador que tiene gusto por la justicia podría estar dispuesto a sacrificar su propio bienestar material, y aportar esfuerzo para castigar al banquero, si percibe que es una persona injusta. Esta extensión es una aplicación de Rabin (1993), en donde los jugadores tienen una función de utilidad que involucra su preferencia por la justicia versus su bienestar material. 
El mecanismo de destrucción de recursos muestra el carácter ex-ante que requiere la regulación a las apuestas de alto riesgo. Dado que la conducta del apostador es difícil de prever, tomando en cuenta una recompensa incierta en status y en vista de que las probabilidades del juego pueden ser percibidas de forma incorrecta, la regulación tiene que dar una solución ex-ante. La regulación financiera en la mayoría de los países trabaja de esta manera, a través de auditorías periódicas y revisiones aleatorias de los libros contables. En otros mercados del tipo de el ganador se lleva todo, donde las apuestas de alto riesgo son posibles, se usa la regulación ex-ante; por ejemplo, la prueba contra el uso de los estimulantes en deportes como un requisito y estrictas reglas de competencia en elecciones políticas.

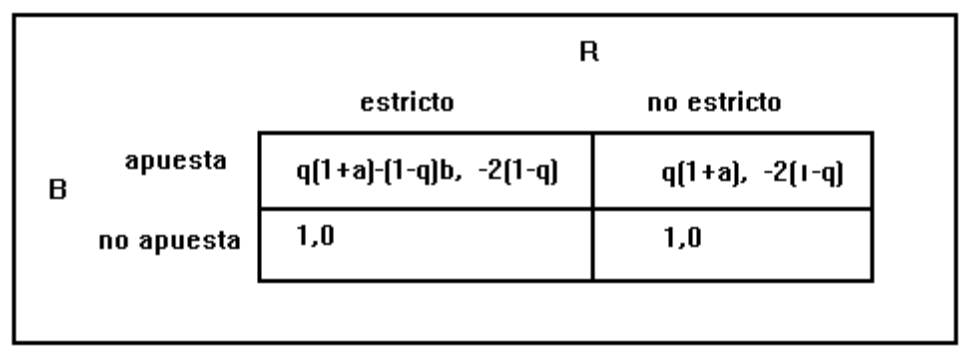

Esquema 3: La apuesta del banquero III 


\section{Discusión}

Dos aspectos que son importantes para la regulación en general, son cruciales en la regulación de las apuestas de alto riesgo: controlar los costos y beneficios del juego, y la creación de un compromiso regulatorio de largo plazo. Primero, en el espíritu de Becker (1968) y Ehrlich (1972), hemos analizado las apuestas de alto riesgo como una decisión económica. Siguiendo esta línea de investigación, debemos cambiar el castigo esperado y la recompensa por apostar, de modo que siempre ofrezcan un pago esperado negativo:

- Reducir a de modo que las apuestas de alto riesgo sean menos rentables, disminuyendo de ese modo la competencia posicional. La reducción podría venir en forma de un tratamiento fiscal especial a las personas con ingresos excepcionales. La misma política fiscal ha sido propuesta por Frank (1985) como una manera de restringir las consideraciones de status y posición social en toma de riesgos.

- Hacer $p$ y $q$ claras y explícitas para todos los jugadores. Se ha encontrado en investigaciones empíricas que los infractores reaccionan más a la probabilidad de ser capturados que a una sanción más alta en caso de ser sorprendidos (ver crimen corporativo en Simpson [1992], evasión fiscal en Klepper y Nagin [1989]). La evidencia muestra que el comportamiento criminal no esta basado en el pago esperado, como en el esquema de Becker, sino que responde a cálculos equivocados de la gente. Es decir, las apuestas de alto riesgo se realizan pensando en ganar. Claramente, el castigo $b$ debe ser conocido, pero el regulador debe enfatizar la probabilidad de obtener un mal resultado - un bajo retorno o ser capturado. La regulación debería tratar de eliminar la tendencia de los apostadores a tener un sesgo en sus probabilidades, a ser optimistas en exceso y a sufrir de disonancia cognoscitiva.

Segundo, el compromiso de la autoridad con el mecanismo establecido es un elemento que se requiere en todas las relaciones legales. El problema de la inconsistencia temporal ha sido analizado anteriormente para otras situaciones económicas: control de inundaciones, políticas de patentes, crédito fiscal a la inversión, e inflación (ver Kydland y Prescott [1977] y Calvo [1978]). Los intereses en el corto y largo plazo de la autoridad también se encuentran en conflicto cuando se enfrenta una apuesta; el regulador quiere disuadir al banquero de apostar, pero si el banquero no apuesta el regulador prefiere no ser estricto. Sin embargo, ser estricto es la única manera de detener la toma de riesgo. Como explicamos en el juego de agente-principal, la manera de resolver este problema económico dinámico es considerar desde el principio el efecto de una regla de política sobre la decisión óptima del agente. Por ello, el regulador debe comprometerse a seguir la regla de ser siempre estricto, sin dejar espacio a la discrecionalidad.

La regla de compromiso explícito no es ningún secreto para la mayoría de los reguladores financieros que realizan un trabajo de largo plazo y tratan de demostrar una rigidez máxima, frente a los agentes que actúan en la mayoría de los casos con un horizonte de más corto plazo. Las autoridades financieras trabajan sobre la base de reglas estrictas y tratan de no establecer relaciones personales con los agentes. Sin embargo, para que este mecanismo funcione, el agente debe estar consciente del carácter del regulador; esto es claro en el esquema de un juego repetido, donde la reputación por sí misma puede resolver este problema de regulación, y por lo tanto es una buena inversión. La reputación es especialmente poderosa cuando la incertidumbre acerca de la naturaleza del regulador se incrementa (ver Kreps y Wilson [1982]). En nuestro juego del regulador y el agente, la naturaleza del regulador es capturada por la incertidumbre acerca de qué tan estricto es.

\section{Conclusiones}

Este documento analizó las apuestas de alto riesgo, particularmente cuando el agente hace una apuesta de todo o nada que causa problemas e impone costos a la economía. La motivación para el jugador no se basa en las consecuencias que sus acciones tengan sobre su ingreso o capacidad de consumo, y es difícil de entender a través del análisis tradicional de la utilidad esperada. La motivación para apostar del jugador es la percepción incorrecta de que tendrá un resultado favorable en su apuesta, y el status que adquirirá al ganar. Se mostró cómo esta conducta de búsqueda de riesgo se explica en psicología, 
sociología y biología como la estrategia del individuo para superar a su grupo. Una tendencia de propensión al riesgo puede ser racional en un mercado donde el ganador se lleva todo, por ejemplo, el mercado financiero. Este análisis esboza una teoría de las apuestas de alto riesgo; más investigación en esta línea deberá tratar de probarla.

Presentamos en este documento, un modelo que muestra a las apuestas de alto riesgo de la forma más simple y poderosa posible. En el mismo esquema, la regulación de la propensión al riesgo del jugador es presentada como un mecanismo de destrucción de recursos. La regulación de un mercado donde el ganador se lleva todo tiene que ser una actividad ex-ante para ser efectiva. Finalmente, la regulación necesita no sólo un castigo claro, sino también un plan de contingencia completo para limitar el costo a la economía en caso que - después de todo - se realice una apuesta de alto riesgo.

El análisis presenta importantes lecciones de política para el regulador financiero. Primero, muestra la motivación que pueden tener los agentes financieros para tomar apuestas de alto riesgo. Segundo, ilustra los incentivos a los que esta sujeto el regulador al supervisar a los intermediarios financieros, de lo que se desprende que su retribución debe depender de la precisión con la que realice su función. Tercero, muestra como un mecanismo de regulación obtiene el resultado deseado sólo cuando las reglas son claras y cuando los beneficios y los castigos son trasparentes ex-ante. Por último, el análisis señala que la acción regulatoria sólo obtendrá la solución óptima cuando elimina cualquier elemento discrecional. 
Anexo 1

La extensión del juego para incorporar la contingencia de que el regulador pueda detectar la apuesta aun cuando ésta haya tenido un resultado bueno se puede lograr con sólo cambiar los supuestos. Consideremos el mismo juego que en el modelo general pero asumamos que cuando el regulador es estricto siempre se percata de las apuestas que se realizan.

En este nuevo juego cuando el regulador es estricto siempre que el banquero apuesta es castigado. Es decir, si el banquero apuesta, la naturaleza elige un buen resultado y el regulador es estricto, entonces el banquero es castigado con un pago de $-b$ (ver esquema IV).

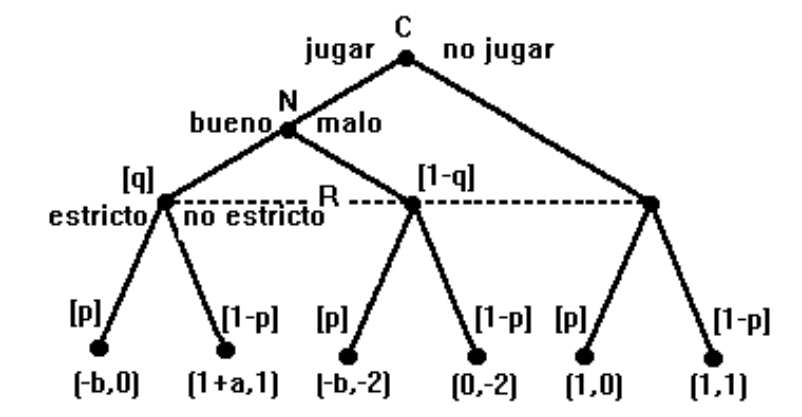

Esquema IV: La apuesta del banquero IV

El mismo juego puede ser expresado en forma estratégica lo que permite ver el resultado de una manera más sencilla (ver esquema $\mathrm{V}$ ). El juego tiene, igual que en el caso general, un solo equilibrio de Nash en (no estricto, apostar), por lo que podemos afirmar que el aspecto cualitativo del juego no cambia. No obstante, si resolvemos el juego con los valores de nuestro ejemplo $\left(q=\frac{1}{\mathbf{4}}, a=4\right.$ y $\left.b=\frac{1}{2}\right)$ tenemos que el resultado cuantitativo es muy diferente. Si el regulador no sigue una estrategia de Nash, la probabilidad con la que necesita ser estricto, para que el banquero no apueste necesita ser sólo mayor a $\frac{1}{7}$. Recordamos que en el modelo general este valor era $\frac{2}{3}$. 
Aunque esta extensión no forma parte central del argumento de este trabajo sí ilustra un punto muy importante para la regulación financiera. Mientras que la decisión entre ser estricto y no estricto es marginal para el resultado del juego, la efectividad en el trabajo del regulador puede cambiar la magnitud del resultado de forma significativa.

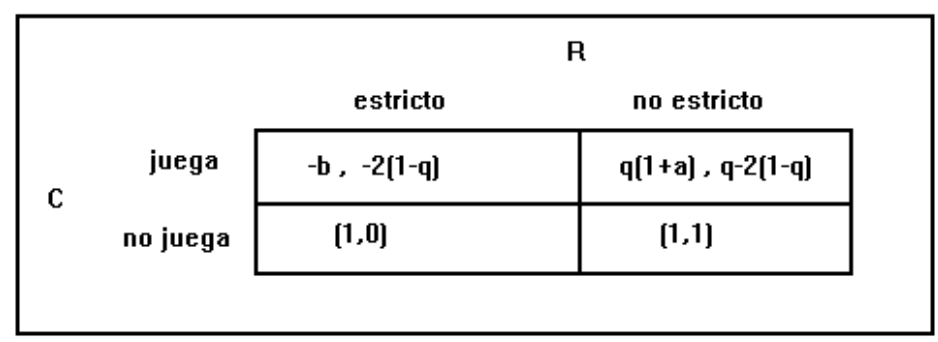

Esquema V: La apuesta del banquero V 


\section{BIBLIOGRAFÍA}

Ainslie, G. 1992. Picoeconomics. Cambridge, MA.: Cambridge University press.

Akerlof, G. y W. Dickens. 1984. "The economic consequences of cognitive dissonance." American Economic Review.

Akerlof, G. 1982. "Labor contracts as partial gift exchange". The Quarterly Journal of Economics.

Allais, M. 1953. "Le comportement de l'homme rationnel devant le risque, critique des postulates et axiomes de l'école americaine". Econométrica.

Arrow, K. 1963. "Social choice and individual values". New Haven: Cowles Foundation.

Asch, P. y R. Quandt. 1987. "Efficiency and profitability in exotic bets." Económica.

Baron, D. y R. Myerson. 1982. "Regulating a monopolist with unknown costs". Econometrica.

Becker, G. 1968. "Crime and punishment: an economic approach." Journal of Political Economy.

Braithwhite, J. 1992. "Poverty power and white-collar crime: Sutherland and the paradoxes of criminological theory." En K. Schlegel y D. Weisburd (eds.), White-Collar Crime Reconsidered. Boston, MA.: Northeastern University Press.

Calvo, G. 1978. "On the time consistency of optimal policy in a monetary economy." Econométrica.

Coleman, J. 1989. The Criminal Elite: the Sociology of White-Collar Crime. New York, NY.: St. Martin's.

Daly, M. y M. Wilson. 1983. Sex, Evolution and Behavior. Boston MA.: PWS Publishers.

Dostoyevsky, F. 1866. The Gambler. U.K.: Everyman.

Ehrlich, I. 1972. "Participation in illegimate activities: a theorical and empirical investigation." Journal of Political Economy.

Frank, R. 1985. Choosing The Right Pond, Human Behavior and the Quest for Status. New York, NY.: Oxford University Press.

Frank, R. y P. Cook. 1992. "Winner-takes-all markets." Cornell University, Mimeo.

Friedman, M. y J. Savage. 1948. "The utility analysis of choices involving risk." Journal of Political Economy.

Geis, G. 1992. "White-collar crime: what is it". En K. Schlegel y D. Weisburd (eds.). White-Collar Crime Reconsidered. Boston, MA.: Northeastern University Press.

Grossman, S. y O. Hart. 1983. "An analysis of the principal-agent problem". Econometrica.

Kahneman, D., P. Slovic y A. Tversky. 1982. Judgement Under Uncertainity: Heuristics and Biases. U.K.: Cambridge University Press.

Kahneman, D. y A. Tversky. 1979. "Prospect theory: an analysis of decision under risk." Econométrica.

Klepper, S. y D. Nagin. 1989. "Tax compliance and perceptions of risks of detection and criminal prosecution." Law and Society Review.

Konner, M. 1990. Why The Reckless Survive. New York, NY.: Viking Penguin.

Kreps, D. y R. Wilson. 1982. "Reputation and imperfect information." Journal of Economic Theory. 
Kydland, F. y E. Prescott. 1977. "Rules rather than discretion: the inconsistency of optimal plans." Journal of Political Economy.

Nisbett, R. y L. Ross. 1980. Human Inference: Strategies and Shorthcomings of Social Judgement. Englewood Cliffs, NJ.: Prentice Hall.

Perloff, L. y B. Fetzer. 1986. "Self-other judgements and perceived vulnerability to victimization." Journal of Personality and Social Psychology.

Rabin, M. 1993. "Incorporating fairness into game theory and economics." American Economic Review.

Romer, P. 1994. "Preferences, promises and the politics of entitlement." University of California, Berkeley, Mimeo.

Rosen, S. 1981. "The economics of superstars." American Economic Review.

Rothschild, M. y Stiglitz J. 1976. "Equilibrium in competitive insurance markets: An essay on the economics of imperfect information". Quarterly Journal of Economics.

Shapiro, S. 1984. Wayward Capitalists: Target of the Securities and Exchange Comission. New Haven, CT.: Yale University Presss.

Simpson, S. 1992. "Corporate crime, deterrance and corporate control policies: views from inside." En K. Schlegel y Weisburd (eds.), White-Collar Crime Reconsidered. Boston, MA.: Northeastern University Press.

Sutherland, E. 1940. "White-collar criminality." American Sociological Review.

Thaler, R y W. Ziemba. 1988. "Parimutuel betting markets: racetrack and lotteries." Journal of Economic Perspectives.

Van Damme, E. 1988. "Stable equilibria and forward induction." Journal of Economic Theory. 